\title{
The Permutable $k$-means for the Bi-partial Criterion
}

Sergey Dvoenko

Tula State University, 92 Lenin Ave., Tula, Russian Federation

E-mail: sergedv@yandex.ru

Jan Owsinski

Systems Research Institute, Polish Academy of Sciences, 6 Newelska, 01447 Warsaw, Poland

E-mail: owsinski@ibspan.waw.pl, http://www.ibspan.waw.pl/glowna/en /

Keywords: distance, similarity, dissimilarity, cluster, $k$-means, objective function

Received: December 18, 2017

The bi-partial criterion for clustering problem consists of two parts, where the first one takes into account intracluster relations, and the second - inter-cluster ones. In the case of k-means algorithm, such bi-partial criterion combines intra-cluster dispersion with inter-cluster similarity, to be jointly minimized. The first part only of such objective function provides the "standard" quality of clustering based on distances between objects (the wellknown classical k-means). To improve the clustering quality based on the bi-partial objective function, we develop the permutable version of k-means algorithm. This paper shows that the permutable k-means appears to be a new type of a clustering procedure.

Povzetek: Študija se ukvarja z gručenjem znotraj in med gručami, pri čemer izvirna metoda uporablja permutirano verzijo običajnega algoritma za gručenje.

\section{Introduction and related works}

\subsection{Clustering by $\boldsymbol{k}$-means}

According to the basic idea of the classical $k$-means algorithm [1-5], a set $\Omega=\left\{\omega_{1}, \ldots \omega_{N}\right\}$ of $N$ elements is divided into clusters $\Omega_{k}, k=1, \ldots K$, represented in a feature space by their "representative" objects $\tilde{\mathbf{x}}_{k}$, and/or "mean" objects $\overline{\mathbf{x}}_{k}$ (centers), where $\mathbf{x}=\left(x_{1}, \ldots x_{n}\right)^{T}$ is a vector in the $n$-dimensional space.

In this paper, we consider means as representatives and calculate new means as in the classical procedure.

The well-known respective clustering criterion minimizes average of squared distances to cluster centers

$$
\begin{gathered}
J(K)=\frac{1}{N} \sum_{k=1}^{K} N_{k} \sigma_{k}^{2}=\sum_{k=1}^{K} \frac{N_{k}}{N} \sigma_{k}^{2}, \\
\sigma_{k}^{2}=\frac{1}{N_{k}} \sum_{i=1}^{N_{k}}\left\|\mathbf{x}_{i}-\overline{\mathbf{x}}_{k}\right\|^{2}=\frac{1}{N_{k}} \sum_{i=1}^{N_{k}} d^{2}\left(\mathbf{x}_{i}, \overline{\mathbf{x}}_{k}\right),
\end{gathered}
$$

where $\sigma_{k}^{2}$ is the dispersion of the cluster $\Omega_{k}$ having size $N_{k}$, and $d(\mathbf{x}, \mathbf{y})$ is the Euclidean distance between vectors $\mathbf{x}$ and $\mathbf{y}$.

As it is well-known [6-10], cluster dispersions can be calculated without direct use of cluster means, based on pairwise distances between vectors

$$
\sigma_{k}^{2}=\frac{1}{2 N_{k}^{2}} \sum_{i=1}^{N_{k}} \sum_{j=1}^{N_{k}}\left\|\mathbf{x}_{i}-\mathbf{x}_{j}\right\|^{2}=\frac{1}{2 N_{k}^{2}} \sum_{i=1}^{N_{k}} \sum_{j=1}^{N_{k}} d^{2}\left(\mathbf{x}_{i}, \mathbf{x}_{j}\right) .
$$

Empirical data often appear in the form of a matrix of pairwise comparisons of elements of the set. Such comparisons can be nonnegative values of dissimilarity or similarity of objects from the set $\Omega$ [11].
This is important for our approach, since the permutable $k$-means, developed in this paper, uses only distance $D(N, N)$ or similarity $S(N, N)$ matrices. Therefore, cluster means are not presented in them, and we need to develop equivalent forms of (1) and (2).

The basis of our approach is the Torgerson's idea of the "gravity center", developed for multidimensional scaling problem [6] in the method of double centering for principal projections to get the appropriate feature space with the raw distance matrix, immersed in it.

Our goal is different: we do not want to restore a feature space itself, since it is sufficient to suppose that objects are immersed in some metric (more closely, Euclidean) space, as we show this later on.

Naturally, the two-component criteria, similar to the bi-partial one (Dunn, Calinski-Harabasz, Xie-Beni etc.), are used in cluster-analysis $[9,12]$. They are mainly used to assess the proper number of clusters $K$. Such criteria are usually heuristic constructions, used to assess the results of some algorithms of quite different origins and properties.

Here we are interested in improving the results of the classical clustering problem with a predefined number of clusters $K$. Namely, we try to develop here the bi-partial objective function to build a homogeneous and strict metric criterion for standard $k$-means algorithm only for a predefined number $K$, and not to use any other idea of procedure than that of $k$-means.

\subsection{The bi-partial criterion}

In order to introduce here a general bi-partial objective function, we refer to an illustrative problem of dividing a 
unidimensional empirical distribution of real values into a set of categories to get the "best" set in a definite sense [13-15]. This case serves merely the purpose of illustration, and assumptions made on data do not apply to the general bi-partial approach.

Let a sequence of $N$ positive real observations $x_{i}, i=1, \ldots N$ be given in non-decreasing order, i.e. with $x_{i+1} \geq x_{i}$ for all of them. Any such sequence can be represented through a cumulative form, obtained via transformation $z_{i}=\sum_{p=1}^{i} x_{p}, i=1, \ldots N$.

As a result, we deal with a convex non-decreasing sequence $z_{i}, i=1, \ldots N$. This means that a straight line, connecting two observations, $z_{q}$ and $z_{s}$, with $1 \leq q<s \leq N$ has all values not under the corresponding observations $z_{i}, i=q, \ldots s$.

Obviously, for the sequence of constant values $x_{1}=\ldots=x_{N}=c$ the convex cumulative form is the line $z_{i}=i c, i=1, \ldots N$, with $z_{1}=c, z_{N}=c N$, represented perfectly by the single linear piece.

Otherwise, for non-constant values, by increasing the number of linear segments from the single one (with $q=1, s=N$ ), we steadily decrease the error of approximation of the original distribution $\left\{z_{i}\right\}$ by the broken line, composed of such segments, down to zero, when the maximal number $N-1$ of linear segments, corresponded to the number of observations $N$, is used to represent the distribution.

Under these conditions, the problem of obtaining the optimal piece-wise linear approximation of the cumulative sequence with the number of linear segments also being optimized was investigated in [13-15].

According to [13-15], the respective bi-partial objective function $J_{D S}$ penalizes, first, deviation $C_{D}$ of linear segments from the respective distribution, and, second, penalizes similarity $C_{S}$ of linear segments to each other, and was represented in the form

$$
J_{D S}(K)=(1-\alpha) C_{D}(K)+\alpha C_{S}(K) \rightarrow \min ,
$$

where $K \geq 1$ is the number of segments, $0 \leq \alpha \leq 1$ is the coefficient of linear combination of two parts of the criterion.

The criterion $J_{D S}$, investigated in [13, 14] for the above problem, is a particular case of the general bi-partial form, representing the fundamental "intra-cluster cohesion + inter-cluster separation" paradigm $[15,16]$.

It should be noted that the parameter $\alpha$ in (3) need not appear at all, if two parts of the objective function are assumed to reflect correctly the respective inner and outer measures. Note that by solving with respect to (3) we get both the cluster (segment) content and the number of clusters (segments). We can also represent (3) in different forms to obtain different data analysis problems as particular cases. So, e.g., (3) can be transformed to the linear regression problem for $K=1, \alpha=0$.

In other interesting cases, the problem (3) can be considered for other kinds of parameters than $\alpha$, say, $K$. Thus, we can treat $K \geq 1$ as a hyper-parameter and find the optimal linear combination of parts in $J_{D S}(K)$.

Thus, in the context of the illustrative problem quoted, we would fix the number of line segments $K$, and look with (3) for the optimum weight $\alpha$, meaning the significance we attach to accuracy of the approximation vs. distinctiveness of the consecutive segments.

In this paper, we investigate the single-parametric reduced form of (3) to find the optimal $\alpha$ for the predefined hyper-parameter $K$ based on the direct implementing of the well-known k-means algorithm.

\section{Distance and similarity $k$-means}

In this paper, we use the specially developed $k$-means algorithm only for the case of distances or similarities between objects $[17,18]$.

A positive definite similarity matrix can be obtained as a matrix of pairwise scalar products of object descriptions in some metric space with the dimensionality of not more than a set cardinal number. This matrix of scalar products can be transformed into a distance matrix and vice versa. As a result, the dissimilarity matrix can be used as the distance matrix in the same space.

In this case, the mean object $\omega\left(\overline{\mathbf{x}}_{k}\right)$ cannot be defined in $\Omega$ by the distance matrix $D(N, N)$ as a center of a cluster. Usually, the object minimizing the sum of distances to the others in the cluster can be used as the center $\bar{\omega}_{k}$. Therefore, if representatives and centers coincide each with other, $\tilde{\omega}_{k}=\bar{\omega}_{k}$ for all clusters, then we get an unbiased clustering.

Nevertheless, if we immerse the set $\Omega$ in some feature space, we obtain in general the biased clustering, since the center $\mathbf{x}\left(\bar{\omega}_{k}\right)$ may not be the mean object $\overline{\mathbf{x}}_{k}$ in the unknown feature space.

The classical $k$-means algorithm was developed for distances and similarities in $[17,18]$. Centers $\bar{\omega}_{k}$ provide the unbiased clustering with cluster dispersions $\sigma_{k}^{2}=\left(1 / N_{k}\right) \sum_{i=1}^{N_{k}} d^{2}\left(\omega_{i}, \bar{\omega}_{k}\right)$ minimizing $J(K)$. If the set $\Omega$ is immersed in a feature space, then two criteria

$$
J^{X}(K)=\min _{\overline{\mathbf{x}}_{1}, \ldots \overline{\mathbf{x}}_{K}} J(K), J^{D}(K)=\min _{\bar{\omega}_{1}, \ldots \bar{\omega}_{K}} J(K)
$$

have not the same values, since $J^{D}(K) \geq J^{X}(K)$ in general. Yet, $J^{X}(K)=J^{D}(K)$, if objects $\mathbf{x}\left(\bar{\omega}_{k}\right)$ and $\overline{\mathbf{x}}_{k}$ are the same.

We would like to guarantee this condition. For some $\omega_{l} \in \Omega$, as a point of the origin and a pair $\omega_{i}, \omega_{j}$, the scalar product is $s_{i j}=\left(d_{l i}^{2}+d_{l j}^{2}-d_{i j}^{2}\right) / 2$, where distance is $d_{p q}=d\left(\omega_{p}, \omega_{q}\right)$ and $s_{i i}=d_{l i}^{2}$ for $i=j$. Therefore, the main diagonal of the matrix $S_{l}(N, N)$ represents the squared distances from the origin $\omega_{l} \in \Omega$ to other objects.

According to [6], it is convenient to put the origin of the feature space in the center of all objects $\omega_{i} \in \Omega, i=1, \ldots N$. Therefore, we put the origin of the 
feature space, cluster by cluster, in each center $\bar{\omega}_{k}$ to represent it by its distances to all other objects in the unknown feature space ( $N_{k}$ is the number of objects in $\Omega_{k}$, $\left.\omega_{p}, \omega_{q} \in \Omega_{k}\right)$ :

$$
d^{2}\left(\omega_{i}, \bar{\omega}_{k}\right)=\frac{1}{N_{k}} \sum_{p=1}^{N_{k}} d_{i p}^{2}-\frac{1}{2 N_{k}^{2}} \sum_{p=1}^{N_{k}} \sum_{q=1}^{N_{k}} d_{p q}^{2},
$$

where, according to (1), (4), the cluster dispersion is

$$
\begin{gathered}
\sigma_{k}^{2}=\frac{1}{N_{k}} \sum_{i=1}^{N_{k}}\left(\frac{1}{N_{k}} \sum_{p=1}^{N_{k}} d_{i p}^{2}-\frac{1}{2 N_{k}^{2}} \sum_{p=1}^{N_{k}} \sum_{q=1}^{N_{k}} d_{p q}^{2}\right)= \\
\frac{1}{2 N_{k}^{2}} \sum_{p=1}^{N_{k}} \sum_{q=1}^{N_{k}} d_{p q}^{2} .
\end{gathered}
$$

Hence, we develop the distance $k$-means algorithm based on the classical principle of the "minimum distance to a cluster center":

(a) Step 0. Determine in some way $K$ centers $\bar{\omega}_{k}^{1}$ and put them as representatives $\tilde{\omega}_{k}^{1}=\bar{\omega}_{k}^{1}, k=1, \ldots K ; s=1$.

Step $s$. Reallocate all objects between clusters:

1. $\omega_{i} \in \Omega_{k}^{s}$, if $d\left(\omega_{i}, \bar{\omega}_{k}^{S}\right) \leq d\left(\omega_{i}, \bar{\omega}_{j}^{s}\right)$ for $\omega_{i} \in \Omega_{j \neq k}^{s}$, $j=1, \ldots K, i=1, \ldots N$.

2. Recalculate centers $\bar{\omega}_{k}^{s}, k=1, \ldots K$, represented by distances $d\left(\omega_{i}, \bar{\omega}_{k}^{s}\right), i=1, \ldots N$.

3. Stop, if $\tilde{\omega}_{k}^{s}=\bar{\omega}_{k}^{s}, k=1, \ldots K$,

else $\tilde{\omega}_{k}^{s+1}=\bar{\omega}_{k}^{s}, \bar{\omega}_{k}^{s+1}=\bar{\omega}_{k}^{s}, k=1, \ldots K$;

$s=s+1$.

Based on the direct recalculation of the criterion (1), the equivalent realization is:

(b) Step 0. Determine in some way $K$ centers $\bar{\omega}_{k}^{1}$ and put them as representatives $\tilde{\omega}_{k}^{1}=\bar{\omega}_{k}^{1}, k=1, \ldots K$; calculate $J^{1}=J^{1}(K)$ and put $\tilde{J}^{1}=\tilde{J}^{1}(K)=J^{1}$ relative to representatives; $s=1$.

Step $s$. Reallocate all objects between clusters:

$$
\begin{aligned}
& \text { 1. } \omega_{i} \in \Omega_{k}^{s} \text {, if } J_{i k}^{S} \leq J_{i p}^{s} \text { for } \omega_{i} \in \Omega_{p \neq k}^{s}, p=1, \ldots K, \\
& i=1, \ldots N \text {. }
\end{aligned}
$$

2. Recalculate centers $\bar{\omega}_{k}^{s}, k=1, \ldots K$, represented by distances $d\left(\omega_{i}, \bar{\omega}_{k}^{s}\right), i=1, \ldots N$; recalculate $J^{s}$.

3. Stop, if $\tilde{J}^{s}=J^{s}$, else $\tilde{J}^{s+1}=J^{s}, J^{s+1}=J^{s}$; $s=s+1$.

A positive definite similarity matrix $S(N, N)$ with elements $s_{i j}=s\left(\omega_{i}, \omega_{j}\right) \geq 0$ can be obtained as a matrix of scalar products in the positive quadrant of the feature space. Relative to some point $\omega_{k} \in \Omega$ as the origin, with $s_{i j}=\left(d_{k i}^{2}+d_{k j}^{2}-d_{i j}^{2}\right) / 2, s_{i i}=d_{k i}^{2}$, distances are defined as $d_{i j}^{2}=s_{i i}+s_{j j}-2 s_{i j}$. The cluster center $\bar{\omega}_{k}$ is represented by its similarities with other objects

$$
s\left(\omega_{i}, \bar{\omega}_{k}\right)=\frac{1}{N_{k}} \sum_{p=1}^{N_{k}} s_{i p}, \omega_{p} \in \Omega_{k}, \omega_{i} \in \Omega, i=1, \ldots N .
$$

The cluster compactness is the mean similarity of the cluster center with respect to other objects (6):

$$
\delta_{k}=\frac{1}{N_{k}} \sum_{i=1}^{N_{k}} s\left(\omega_{i}, \bar{\omega}_{k}\right)=\frac{1}{N_{k}^{2}} \sum_{i=1}^{N_{k}} \sum_{p=1}^{N_{k}} s_{i p} ; \omega_{i}, \omega_{p} \in \Omega_{k} .
$$

The unbiased clustering minimizes the cluster dispersion $\sigma_{k}^{2}$ and maximizes the compactness $\delta_{k}$ according to (5):

$$
\sigma_{k}^{2}=\frac{1}{2 N_{k}^{2}} \sum_{i=1}^{N_{k}} \sum_{j=1}^{N_{k}}\left(s_{i i}+s_{j j}-2 s_{i j}\right)=\frac{1}{N_{k}} \sum_{i=1}^{N_{k}} s_{i i}-\delta_{k},
$$

and for all clusters:

$$
J(K)=\sum_{k=1}^{K} \frac{N_{k}}{N} \sigma_{k}^{2}=\frac{1}{N} \sum_{i=1}^{N} s_{i i}-\sum_{k=1}^{K} \frac{N_{k}}{N} \delta_{k}=C-I(K) .
$$

For similarity clustering, we maximize compactness $I(K)$, with $I(K)=C-J(K)$. The similarity $k$-means algorithm is the analogue of algorithms (a) and (b) relative to $I(K)$.

\section{The bi-partial criterion for clustering}

In this paper, we develop the bi-partial objective function like (3) for the dissimilarity $k$-means

$$
J_{\delta}(K)=(1-\alpha) J(K)+\alpha \delta(K),
$$

so as to combine $J(K)$ for intra-cluster distances with the inter-cluster similarity $\delta(K)$. We define the inter-cluster similarity $\delta(K)=(1 / K) \sum_{k=1}^{K} s\left(\bar{\omega}_{k}, \bar{\omega}_{0}\right)$ relative to the center of the whole set, being the object $\bar{\omega}_{0}$, represented by its similarities with respect to all other centers

$$
\begin{gathered}
s\left(\bar{\omega}_{k}, \bar{\omega}_{0}\right)=(1 / K) \sum_{p=1}^{K} s\left(\bar{\omega}_{k}, \bar{\omega}_{p}\right) ; \bar{\omega}_{k}, k=1, \ldots K: \\
\delta(K)=\frac{1}{K^{2}} \sum_{k=1}^{K} \sum_{l=1}^{K} s\left(\bar{\omega}_{k}, \bar{\omega}_{l}\right) .
\end{gathered}
$$

Unfortunately, the bi-partial criterion $J_{\delta}(K)$, as defined here, does not work for the classical $k$-means (b), since (8), as the second part of $J_{\delta}(K)$ in (7), cannot be changed for constant centers while attempting to transfer objects in step $s$.

Therefore, for any $0 \leq \alpha<1$, the clustering results are the same as for the classical case with $\alpha=0$. And the algorithm does not work properly with $\alpha=1$.

We develop here the new "permutable" version of the classical $k$-means (b) without direct calculation of cluster centers. Here, the new permutable $k$-means is the meanless clustering for the classical $k$-means (b).

As we can see in (5), the cluster dispersion is half of the average of squared distances between objects in the cluster. This representation does not contain centers themselves, and we calculate the criterion (1) without centers in the form

$$
J(K)=\sum_{k=1}^{K} \frac{N_{k}}{N} \sigma_{k}^{2}=\frac{1}{2 N} \sum_{k=1}^{K} \frac{1}{N_{k}} \sum_{p=1}^{N_{k}} \sum_{q=1}^{N_{k}} d_{p q}^{2} .
$$

Next, we would like to calculate the similarity $s\left(\bar{\omega}_{k}, \bar{\omega}_{l}\right)$ between cluster centers in (8). According to (6), 
the average similarity of the center $\bar{\omega}_{k}$ with the objects from the other cluster $\omega_{i} \in \Omega_{l}$ is

$$
s\left(\Omega_{l}, \bar{\omega}_{k}\right)=\frac{1}{N_{l}} \sum_{i=1}^{N_{l}} s\left(\omega_{i}, \bar{\omega}_{k}\right)=\frac{1}{N_{l} N_{k}} \sum_{i=1}^{N_{l}} \sum_{p=1}^{N_{k}} s_{i p}, \omega_{p} \in \Omega_{k} .
$$

It is evident that $s\left(\Omega_{l}, \bar{\omega}_{k}\right)=s\left(\Omega_{k}, \bar{\omega}_{l}\right)$, as $s_{i j}=s_{j i}$. Therefore, we can use the suitable notation $s\left(\Omega_{l}, \bar{\omega}_{k}\right)=s\left(\Omega_{k}, \bar{\omega}_{l}\right)=s\left(\Omega_{l}, \Omega_{k}\right)=s\left(\bar{\omega}_{l}, \bar{\omega}_{k}\right)$. Hence, (8) is converted into $\left(\omega_{p} \in \Omega_{k}, \omega_{q} \in \Omega_{l}\right)$ :

$$
\delta(K)=\frac{1}{K^{2}} \sum_{k=1}^{K} \sum_{l=1}^{K} \frac{1}{N_{k} N_{l}} \sum_{p=1}^{N_{k}} \sum_{q=1}^{N_{l}} s_{p q} .
$$

The goal of $J_{\delta}(K)$ is to produce clusters with possibly low dispersion and possibly dissimilar centers. We note that (10) is in a way an inconsistent function, since for $k=l$ it contains the cluster compactness $\delta_{k}$. Hence, we modify (10) to get the inter-cluster similarities only and take into account the symmetry

$$
\delta(K)=\frac{1}{2 K(K-1)} \sum_{k=1}^{K} \sum_{l=1, l \neq k}^{K} \frac{1}{N_{k} N_{l}} \sum_{p=1}^{N_{k}} \sum_{q=1}^{N_{l}} s_{p q},
$$

for $\omega_{p} \in \Omega_{k}, \omega_{q} \in \Omega_{l}$.

We develop here the classical $k$-means (b) in the new form of the permutable $k$-means based on (9)-(11):

(c) Step 0. Determine in some way the sets $\Omega_{l}^{1}, l=1, \ldots K$; define $\alpha$, calculate $J^{1}=J_{\delta}^{1}(K) ; s=1$.

Step $s$. Reallocate all objects between clusters:

1. Remember, but do not move: $\omega_{i} \in \Omega_{k}^{s}$, if $J_{i k}^{s} \leq J_{i p}^{s}$ for $\omega_{i} \in \Omega_{p \neq k}^{s}, p=1, \ldots K, i=1, \ldots N$.

2. Reallocate all objects $\omega_{i}, i=1, \ldots N$ at once; calculate $J^{s+1}$.

3. If $J^{s+1}=J^{s}$ then stop;

If $J^{s+1}>J^{s}$ then: cancel last reallocations, $J^{s+1}=J^{s}$, stop;

If $J^{s+1}<J^{s}$ then: $J^{s+1}=J^{s}, s=s+1$.

As we can see, in the step ( $s .1)$ we recalculate the criterion $J^{s}$ in order to get its modified value $J_{i p}^{s}$. Let $\omega_{i} \in \Omega_{j}^{s}$. When trying to move $\omega_{i}$ from $\Omega_{j}^{s}$ to some other $\Omega_{p}^{s}$, we try to change the respective sets to $\Omega_{j}^{s} \backslash \omega_{i}$ and to $\Omega_{p}^{s} \cup \omega_{i}$. Changes in the sets result in implicit changes of their centers, even though we do not calculate them. Consequently, this action differs from the same one in algorithms (a) and (b) for constant centers.

Algorithm (c) appears to be a new type of clustering procedures, since its result differs, in general, from those of the classical (a) and (b) procedures, both for the classical $(\alpha=0)$ and the proper bi-partial $(\alpha>0)$ cases. In addition, we can use some optimal initial clusters to enhance the quality of results, and optimal recalculations to improve performance of permutations.

As we can see, the algorithm (c) is the same as the classical ones (a) and (b) for the standard criterion $J(K)$ and differs (sometimes subtly and finely) from them for the bi- partial criterion $J_{\delta}(K)$.

It is clear that the new algorithm gives the classical result for non-intersecting clusters. Nevertheless, its result can be improved for intersecting clusters, since by means of the criterion $J_{\delta}(K)$ a cluster center can be shifted in some vicinity without changing the cluster itself. Such possibility depends on the gaps between real points in continuous feature space and the discrete cluster structure superimposed.

\section{Redistribution of data dispersion by the bi-partial criterion}

Here, we explain why by means of the criterion (7) it is possible to improve the classical clustering of $k$-means.

Consider the classical case. Let the set of size $N$ be divided into $K$ subsets (clusters). In our perspective, we consider balancing of total dispersion between its intraand inter- parts. We know $[19,20]$ that $S_{T}=S_{W}+S_{B}$, where $S_{T}$ is the total scatter matrix, $S_{W}$ is the intra-cluster and $S_{B}$ is the inter-cluster scatter matrices. Therefore, $t r S_{T}=t r S_{W}+t r S_{B}$ for diagonal elements only. Since $\operatorname{tr} S_{T}=N \sigma_{T}^{2}, \operatorname{tr} S_{W}=N \sigma_{W}^{2}$, and $\operatorname{tr} S_{B}=N \sigma_{B}^{2}$, then finally $\sigma_{T}^{2}=\sigma_{W}^{2}+\sigma_{B}^{2}$.

Let the set $\Omega=\left\{\omega_{1}, \ldots \omega_{N}\right\}$ be immersed in some metric space and represented by the distance matrix $D(N, N)$ only with elements $d_{i j}=d\left(\omega_{i}, \omega_{j}\right) \geq 0$. Let $\Omega$ be split into groups $\Omega_{k}, k=1, \ldots K$. Based on the Torgerson's formula, we define the following:

for single group dispersions

$$
\sigma_{k}^{2}=\frac{1}{2 N_{k}^{2}} \sum_{p=1}^{N_{k}} \sum_{q=1}^{N_{k}} d^{2}\left(\omega_{p}, \omega_{q}\right), k=1, \ldots K ;
$$

for the intra-group dispersion

$$
\begin{gathered}
\sigma_{W}^{2}=\sum_{k=1}^{K} \frac{N_{k}}{N} \sigma_{k}^{2}=\sum_{k=1}^{K} \frac{N_{k}}{N} \frac{1}{2 N_{k}^{2}} \sum_{p=1}^{N_{k}} \sum_{q=1}^{N_{k}} d^{2}\left(\omega_{p}, \omega_{q}\right)= \\
\frac{1}{2 N} \sum_{k=1}^{K} \frac{1}{N_{k}} \sum_{p=1}^{N_{k}} \sum_{q=1}^{N_{k}} d^{2}\left(\omega_{p}, \omega_{q}\right) ;
\end{gathered}
$$

for the total dispersion

$$
\begin{gathered}
\sigma_{T}^{2}=\frac{1}{2 N^{2}} \sum_{p=1}^{N} \sum_{q=1}^{N} d^{2}\left(\omega_{p}, \omega_{q}\right)= \\
\frac{1}{2 N^{2}} \sum_{k=1}^{K} \sum_{l=1}^{K} \sum_{p=1}^{N_{k}} \sum_{q=1}^{N_{l}} d^{2}\left(\omega_{p}, \omega_{q}\right) ;
\end{gathered}
$$

for the inter-center dispersion

$$
\sigma_{I C}^{2}=\frac{1}{K} \sum_{k=1}^{K} d^{2}\left(\bar{\omega}_{k}, \bar{\omega}_{0}\right)=\frac{1}{2 K^{2}} \sum_{p=1}^{K} \sum_{q=1}^{K} d^{2}\left(\bar{\omega}_{p}, \bar{\omega}_{q}\right),
$$

where the center $\bar{\omega}_{0}$ of the set $\Omega$ is represented by its distances to other centers $\bar{\omega}_{k}$ through

$$
d^{2}\left(\bar{\omega}_{k}, \bar{\omega}_{0}\right)=\frac{1}{K} \sum_{p=1}^{K} d^{2}\left(\bar{\omega}_{k}, \bar{\omega}_{p}\right)-\sigma_{I C}^{2} .
$$

We remark that the classical inter-group dispersion is not given by the Torgerson's formula 


$$
\sigma_{B}^{2}=\sum_{k=1}^{K} \frac{N_{k}}{N} d^{2}\left(\bar{\omega}_{k}, \bar{\omega}_{0}\right) .
$$

Therefore, the classical inter-group dispersion is

$$
\begin{gathered}
\sigma_{B}^{2}=\sum_{k=1}^{K} \frac{N_{k}}{N}\left(\frac{1}{K} \sum_{p=1}^{K} d^{2}\left(\bar{\omega}_{k}, \bar{\omega}_{p}\right)-\sigma_{I C}^{2}\right)= \\
\frac{1}{K} \sum_{k=1}^{K} \frac{N_{k}}{N} \sum_{p=1}^{K} d^{2}\left(\bar{\omega}_{k}, \bar{\omega}_{p}\right)-\sigma_{I C}^{2} \sum_{p=1}^{K} \frac{N_{k}}{N}= \\
\frac{1}{K} \sum_{k=1}^{K} \frac{N_{k}}{N} \sum_{p=1}^{K} d^{2}\left(\bar{\omega}_{k}, \bar{\omega}_{p}\right)-\sigma_{I C}^{2} .
\end{gathered}
$$

As shown above, we minimize the classical criterion $J(K)$ based on the distance matrix $D(N, N)$, and maximize the criterion in the dual form $I(K)=C-J(K)$ based on the similarity matrix $S(N, N)$.

Hence, in the dual form of the bi-partial criterion we try to maximize the classical part $I(K)$ and the new second part for the inter-center dispersion $\sigma_{I C}^{2}$, as based on the Torgerson's formula. Since the classical inter-group dispersion $\sigma_{B}^{2}$ is not based on the Torgerson's formula, we calculate it with distances $d^{2}\left(\bar{\omega}_{k}, \bar{\omega}_{0}\right)$. Such distances refer to distances between sets, not being a topic here.

Hence, in the dual form by maximizing $I(K)$, we minimize strictly equivalent classical $J(K)$ and maximize the inter-center dispersion $\sigma_{I C}^{2}$. Since $\sigma_{T}^{2}=\sigma_{W}^{2}+\sigma_{B}^{2}$, we have the decomposition

$$
\sigma_{T}^{2}=\sigma_{W}^{2}+\frac{1}{K} \sum_{k=1}^{K} \frac{N_{k}}{N} \sum_{p=1}^{K} d^{2}\left(\bar{\omega}_{k}, \bar{\omega}_{p}\right)-\sigma_{I C}^{2} .
$$

Let us denote $\sigma_{B \cup I C}^{2}=\frac{1}{K} \sum_{k=1}^{K} \frac{N_{k}}{N} \sum_{p=1}^{K} d^{2}\left(\bar{\omega}_{k}, \bar{\omega}_{p}\right)$ and represent the classical inter-group dispersion in the form $\sigma_{B}^{2}=\sigma_{B \cup I C}^{2}-\sigma_{I C}^{2}$ without the contribution of the intercenter dispersion, where $\sigma_{T}^{2}+\sigma_{I C}^{2}=\sigma_{W}^{2}+\sigma_{B \cup I C}^{2}$.

As we can see, the permutable $k$-means is targeted to minimize $J(K)=\sigma_{W}^{2}$. Since the total dispersion $\sigma_{T}^{2}=$ const , at the same time the classical inter-group dispersion $\sigma_{B}^{2}=\sigma_{B \cup I C}^{2}-\sigma_{I C}^{2}$ is maximized. Therefore, the balance $\sigma_{T}^{2}=\sigma_{W}^{2}+\sigma_{B}^{2}$ remains true. The decomposition $\sigma_{T}^{2}+\sigma_{I C}^{2}=\sigma_{W}^{2}+\sigma_{B \cup I C}^{2}$ shows that the balance of two parts is maintained, while we increase both of them.

In this case, the bi-partial criterion influences $\sigma_{I C}^{2}$ only. Hence, by means of the bi-partial criterion we manipulate to maximize the inter-center dispersion $\sigma_{I C}^{2}$ with the other part $\sigma_{B \cup I C}^{2}$ being maximized "as is".

\section{Experiments}

\subsection{Experimental setup}

Experimental data are the original Fisher's Iris data [21]. We chose this data set as a simple illustration for the basic properties of the approach developed. Such data consist of 150 measurements of 50 plants, belonging to three varieties: Iris setosa, Iris versicolor, and Iris virginica. Four flower measurements are made: petal length and width, and sepal length and width.

It is known that the $1^{\text {st }}$ class (Iris setosa) is well separated from other two classes ( $2^{\text {nd }}$ class, Iris versicolor, and $3^{\text {rd }}$ class, Iris virginica). The $2^{\text {nd }}$ and $3^{\text {rd }}$ classes intersect each other. Another peculiarity of Iris data is the coincidence of objects 102 and 143 from the $3^{\text {rd }}$ class. Iris data are also included in Matlab.

There are also other available variants of the Iris data, differing from the classic set of [21]. Such differences usually concern corrections in some measurements.

Since the classification of data has been defined, we show that the bi-partial objective function $J_{\delta}(K)$, developed above, allows us to improve the classical clustering result. According to it, we separate as usual $1^{\text {st }}$ class correctly from two others, and decrease the errors in separation of the $2^{\text {nd }}$ and the $3^{\text {rd }}$ class.

According to the formulation above, we investigate the problem

$$
\alpha^{*}=\underset{0 \leq \alpha \leq 1}{\arg \min } J_{\delta}(K)=\underset{0 \leq \alpha \leq 1}{\arg \min }((1-\alpha) J(K)+\alpha \delta(K)) .
$$

As we can see, this formulation implies balancing of two parts of the criterion. Therefore, it would be good to measure $J(K)$ and $\delta(K)$ on the same scale.

The dispersion of standardized data is $n$, i.e. the number of features ( $n=4$ for Iris data), and usually more than $n$ for original (non-standardized) data. The clustering results for original and standardized data can differ.

In order to get rid of the potential scale bias, we normalize inter-cluster similarities $s_{k l}^{\prime}=s_{k l} / \sqrt{s_{k k} s_{l l}}$ to get $s_{k k}^{\prime}=1,0 \leq s_{k l}^{\prime} \leq 1 ; k, l=1, \ldots K$.

The last technical remark regarding the correctness of the criterion $J_{\delta}(K)$ is that in the case of usual standard multidimensional data, we need to move the origin out of the convex cover of the set relative to its center and provide positive scalar products as similarities between objects. This problem was discussed in [22].

Indeed, as it is mentioned above, all similarities in (6), (8), (10), (11) must be nonnegative for correct $I(K)$ and $\delta(K)$. According to (4), the origin is placed in the center of the data set in the feature space.

Unfortunately, it this case we cannot use scalar products $s_{i j}=\left(d_{k i}^{2}+d_{k j}^{2}-d_{i j}^{2}\right) / 2$ in $J_{\delta}(K)$, since they can have negative values. Nevertheless, scalar products change to nonnegative values with respect to the origin placed out of the convex cover of the set, since all of them appear to be in the positive quadrant of the feature space. Hence, it does not matter at all for distances (they have been calculated and not changed for any place of the origin), but it is correct to represent nonnegative similarities by scalar products.

It is known that the $k$-means algorithm is the locally optimal procedure with results dependent on initial decisions (partition or choice of centers).

For all classes, we test three initial partitions: 50/50/50 
(plant varieties as classes), 50/70/30 (20 plants from the $3^{\text {rd }}$ class are wrongly placed in the $2^{\text {nd }}$ class $), 50 / 30 / 70$ (20 plants from the $2^{\text {nd }}$ class are wrongly placed in the $3^{\text {rd }}$ class).

For just two intersecting classes $\left(2^{\text {nd }}\right.$ and $\left.3^{\text {rd }}\right)$ we test also three initial partitions: 50/50 (plant varieties as classes), 70/30 (20 plants from the $3^{\text {rd }}$ class are wrongly placed in the $2^{\text {nd }}$ class), $30 / 70$ (20 plants from the $2^{\text {nd }}$ class are wrongly placed in the $3^{\text {rd }}$ class).

In yet another case we investigate two classes of the entire set, organized as the small one ( $1^{\text {st }}$ class) and the big one ( $2^{\text {nd }}$ and $3^{\text {rd }}$ classes). We test three initial partitions: $50 / 100$ (plants from the $1^{\text {st }}$ class versus all plants from the $2^{\text {nd }}$ and $3^{\text {rd }}$ classes together), $100 / 50$ (all plants from the $1^{\text {st }}$ and the $2^{\text {nd }}$ classes together versus plants from the $3^{\text {rd }}$ class), 30/120 (only first 30 plants from the $1^{\text {st }}$ class versus all others).

In all experiments, we first get the classical result with $\alpha=0$, starting from the predefined initial partitions as above. Second, starting, as well, from the predefined initial partitions characterized above, we vary the parameter $0<\alpha \leq 1$ with increment 0.01 to find the optimal $\alpha^{*}$ among the tested 100 points.

\subsection{Results and discussion}

In the first experiment with original Iris data for all initial partitions for three classes, we correctly separate the $1^{\text {st }}$ class and decrease errors in separation of intersecting $2^{\text {nd }}$ and $3^{\text {rd }}$ classes (Table 1, Fig.1). For two intersecting classes only, we decrease errors in the separation of the $2^{\text {nd }}$ and $3^{\text {rd }}$ classes, too (Table 1, Fig. 2, 3). It can be seen that the optimal intervals for $\alpha^{*}$ depend on the number of clusters (Table 1), hence on data dispersion, and can slightly differ for different initial partitions. Error diagrams are not monotonic functions (Fig. 1-3).

As we can see, original Iris data are some sort of "well structured" data, since for different initial partitions we get the same 16 misclassified objects for the classical $(\alpha=0)$ criterion and the same 15 misclassified objects for the bipartial $\left(\alpha^{*}\right)$ criterion (Table 1). For the classical criterion, misclassified objects are generally from the $3^{\text {rd }}$ class (Table 2). The object 135 is well classified and shown here, since it is misclassified for the bi-partial criterion.

Misclassified objects for the bi-partial criterion are from the $3^{\text {rd }}$ class, too (Table 3 ). Here, objects 53 and 78 are well classified, and the object 135 is misclassified.

We repeat this experiment for standardized data (Table 4). Such data are more complicated. As we know, Iris classes are not so spherical ones in the original feature space, and that is why the $k$-means type of approach is not the best suited for them.

After data standardization, classes appear to be more spherical and contain more "mixed" objects from intersecting classes, usually giving more misclassifications in the classical case (Table 4).

Hence, for the classical criterion $(\alpha=0)$ for standardized data, 25 misclassified objects are from two intersecting classes, $2^{\text {nd }}$ and $3^{\text {rd }}$, with well classified all objects from the $1^{\text {st }}$ class (Table 5). Objects 104, 109, 112, 126,
129 are well classified and shown too, since they are misclassified for the bi-partial criterion.

Misclassified objects for the bi-partial criterion are mainly from the $3^{\text {rd }}$ class again (Table 6 ). Here, objects 52 , $57,66,71,76,86,87$ are well classified and objects 104, $109,112,128,129$ are misclassified.

Table 1: Clustering results of original Iris data.

\begin{tabular}{|c|c|c|c|c|}
\hline $\begin{array}{c}\text { Initial } \\
\text { partitions }\end{array}$ & $\begin{array}{c}\text { Errors } \\
(\alpha=0)\end{array}$ & $\alpha^{*}$ & $\begin{array}{c}\text { Errors } \\
\left(\alpha^{*}\right)\end{array}$ & Diagrams \\
\hline $50 / 50 / 50$ & 16 & $0.6 \div 0.75$ & 15 & \multirow{2}{*}{ Fig. 1} \\
\hline $50 / 70 / 30$ & 16 & $0.6 \div 0.75$ & 15 & \\
\hline $50 / 30 / 70$ & 16 & $0.6 \div 0.75$ & 15 & \\
\hline $50 / 50$ & 16 & $0.81 \div 0.92$ & 15 & \multirow{2}{*}{ Fig. 2} \\
\hline $70 / 30$ & 16 & $0.81 \div 0.92$ & 15 & \\
\hline $30 / 70$ & 16 & $0.81 \div 0.91$ & 15 & Fig. 3 \\
\hline
\end{tabular}

Table 2: Classical 16 misclassifications of original Iris data.

\begin{tabular}{|c|c|c|}
\hline$\alpha=0$ & & \\
$50 / 50 / 50$ 50/50 & $2^{\text {nd }}$ cluster & $3^{\text {rd }}$ cluster \\
$50 / 70 / 3070 / 30$ & & \\
$50 / 30 / 7030 / 70$ & & 53 \\
\hline \multirow{2}{*}{ Iris versicolor } & & 78 \\
$2^{\text {nd }}$ class (51-100) & & \\
\hline & 102120128147 & \\
Iris virginica & 107122134150 & Correct: 135 \\
$3^{\text {rd }}$ class (101-150) & 114124139 & \\
& 115127143 & \\
\hline
\end{tabular}

Table 3: Bi-partial 15 misclassifications of original Iris data.

\begin{tabular}{|c|c|c|}
\hline$\alpha^{*}$ & & \\
$50 / 50 / 50$ 50/50 & $2^{\text {nd }}$ cluster & $3^{\text {rd }}$ cluster \\
$50 / 70 / 3070 / 30$ & & \\
$50 / 30 / 7030 / 70$ & $\mathbf{5 3}$ & \\
\hline Iris versicolor & $\mathbf{7 8}$ & \\
$2^{\text {nd }}$ class (51-100) & 102120128147 & \\
& 107122134150 & \\
Iris virginica & 114124139 & \\
$3^{\text {rd }}$ class (101-150) & 115127143 & \\
& $\mathbf{1 3 5}$ & \\
\hline
\end{tabular}

Table 4: Clustering results of standardized Iris data.

\begin{tabular}{|c|c|c|c|c|}
\hline $\begin{array}{c}\text { Initial } \\
\text { partitions }\end{array}$ & $\begin{array}{c}\text { Errors } \\
(\alpha=0)\end{array}$ & $\alpha^{*}$ & $\begin{array}{c}\text { Errors } \\
\left(\alpha^{*}\right)\end{array}$ & Diagrams \\
\hline $50 / 50 / 50$ & 25 & 0.85 & 22 & \multirow{2}{*}{ Fig. 4 } \\
\hline $50 / 70 / 30$ & 25 & 0.85 & 22 & \\
\hline $50 / 30 / 70$ & 25 & 0.85 & 22 & \\
\hline $50 / 50$ & 17 & $0.94 \div 0.97$ & 15 & Fig. 5 \\
\hline $70 / 30$ & 17 & $0.92 \div 0.97$ & 15 & Fig. 6 \\
\hline $30 / 70$ & 17 & $0.83 \div 0.95$ & 14 & Fig. 7 \\
\hline
\end{tabular}


Table 5: Classical 25 misclassifications of standardized Iris data.

\begin{tabular}{|c|l|l|}
\hline $\begin{array}{c}\alpha=0 \\
50 / 50 / 50 \\
50 / 70 / 30\end{array}$ & \multicolumn{1}{|l|}{$2^{\text {nd }}$ cluster } & $3^{\text {rd }}$ cluster \\
$50 / 30 / 70$ & & \\
\hline Iris versicolor & & 51577686 \\
$2^{\text {nd }}$ class (51-100) & & 52667787 \\
& & 537178 \\
\hline & 102120134147 & Correct: \\
& 107122135150 & 129 \\
Iris virginica & 114124139 & 112 \\
$3^{\text {rd }}$ class (101-150) & 115127143 & 128 \\
& & \\
\hline
\end{tabular}

Table 6: Bi-partial 22 misclassifications of standardized Iris data.

\begin{tabular}{|c|c|c|}
\hline $\begin{array}{c}\alpha^{*} \\
50 / 50 / 50 \\
50 / 70 / 30 \\
50 / 30 / 70 \\
\end{array}$ & $2^{\text {nd }}$ cluster & $3^{\text {rd }}$ cluster \\
\hline $\begin{array}{c}\text { Iris versicolor } \\
2^{\text {nd }} \text { class }(51-100)\end{array}$ & $\begin{array}{l}527186 \\
577687 \\
6677 \\
\end{array}$ & $\begin{array}{l}51 \\
53 \\
78 \\
\end{array}$ \\
\hline $\begin{array}{c}\text { Iris virginica } \\
3^{\text {rd }} \text { class }(101-150)\end{array}$ & $\begin{array}{llll}102 & 122 & 139 & \mathbf{1 0 4} \\
107 & 124 & 143 & \mathbf{1 0 9} \\
114 & 127 & 147 & \mathbf{1 1 2} \\
115 & 134 & 150 & \mathbf{1 2 8} \\
120 & 135 & & \mathbf{1 2 9}\end{array}$ & \\
\hline
\end{tabular}

Table 7: Classical 17 misclassifications of standardized Iris data.

\begin{tabular}{|c|c|c|c|}
\hline \multicolumn{2}{|r|}{$\alpha=0$} & $2^{\text {nd }}$ cluster & $3^{\text {rd }}$ cluster \\
\hline \multirow[b]{2}{*}{$50 / 50$} & $\begin{array}{c}\text { Iris versicolor } \\
2^{\text {nd }} \text { class } \\
(51-100)\end{array}$ & & $\begin{array}{l}51 \\
53 \\
78 \\
\end{array}$ \\
\hline & $\begin{array}{c}\text { Iris virginica } \\
3^{\text {rd }} \text { class } \\
(101-150)\end{array}$ & $\begin{array}{llll}102 & 122 & 134 \\
147 & & \\
107 & 124 & 135 \\
150 & & \\
114 & 127 & 139 \\
120 & 128 & 143 \\
\end{array}$ & Correct: 112 \\
\hline \multirow[b]{2}{*}{$70 / 30$} & $\begin{array}{c}\text { Iris versicolor } \\
2^{\text {nd }} \text { class } \\
(51-100)\end{array}$ & & $\begin{array}{l}51 \\
53 \\
78 \\
\end{array}$ \\
\hline & $\begin{array}{c}\text { Iris virginica } \\
3^{\text {rd }} \text { class } \\
(101-150)\end{array}$ & $\begin{array}{llll}102 & 122 & 134 \\
147 & & \\
107 & 124 & 135 \\
150 & & \\
114 & 127 & 139 \\
120 & 128 & 143 \\
\end{array}$ & Correct: 112 \\
\hline \multirow[b]{2}{*}{$30 / 70$} & $\begin{array}{c}\text { Iris versicolor } \\
2^{\text {nd }} \text { class } \\
(51-100)\end{array}$ & $\begin{array}{l}\text { Correct: } \\
527187 \\
5777 \\
6686\end{array}$ & $\begin{array}{l}51 \\
53 \\
78\end{array}$ \\
\hline & $\begin{array}{c}\text { Iris virginica } \\
3^{\text {rd }} \text { class } \\
(101-150)\end{array}$ & $\begin{array}{llll}102 & 122 & 134 \\
147 & & \\
107 & 124 & 135 \\
150 & & \\
114 & 127 & 139 \\
120 & 128 & 143\end{array}$ & \\
\hline
\end{tabular}




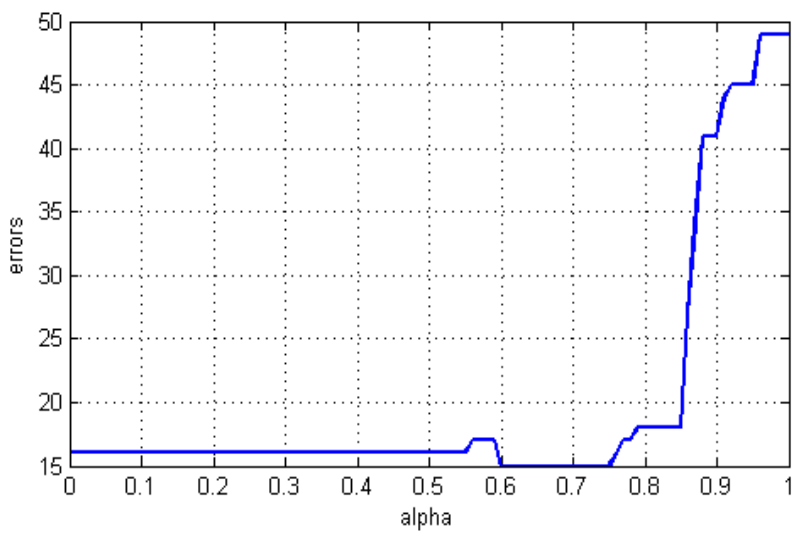

Figure 1. Clustering errors of original Iris data for Setosa/Versicolor/Virginica varieties (50/50/50, 50/70/30, $50 / 30 / 70$ ) with 15 misclassified objects.

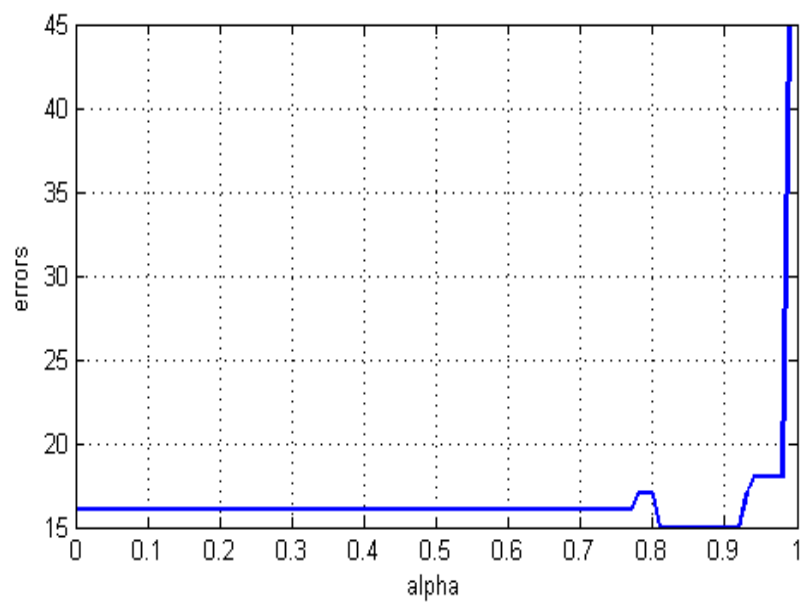

Figure 2. Clustering errors of original Iris data for Versicolor/Virginica varieties $(50 / 50,70 / 30)$ with 15 misclassified objects.

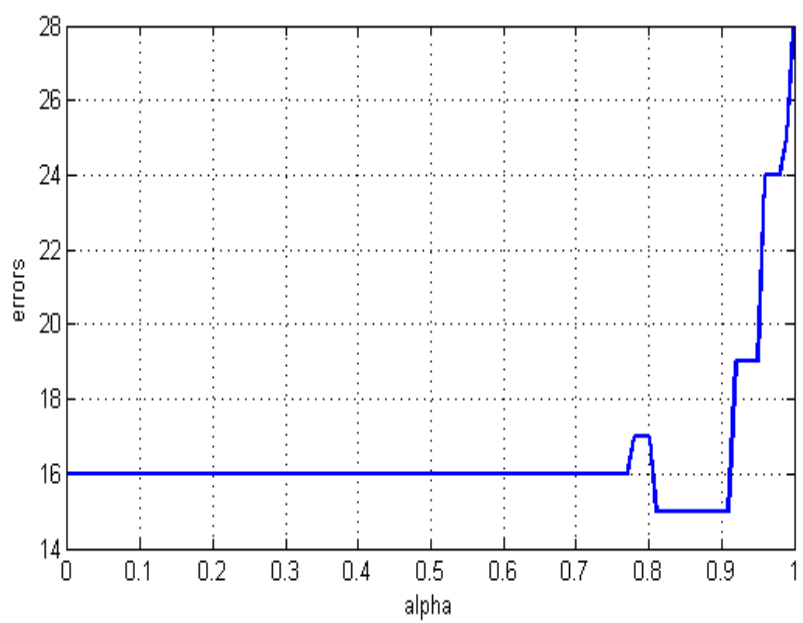

Figure 3. Clustering errors of original Iris data for Versicolor/Virginica varieties (30/70) with 15 misclassified objects.

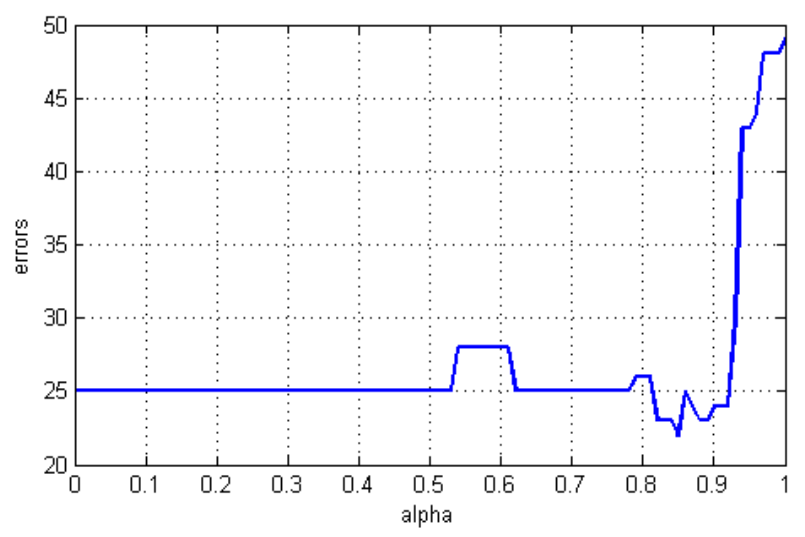

Figure 4. Clustering errors of standardized Iris data for Setosa/Versicolor/Virginica varieties (50/50/50, 50/70/30, $50 / 30 / 70$ ) with 22 misclassified objects.

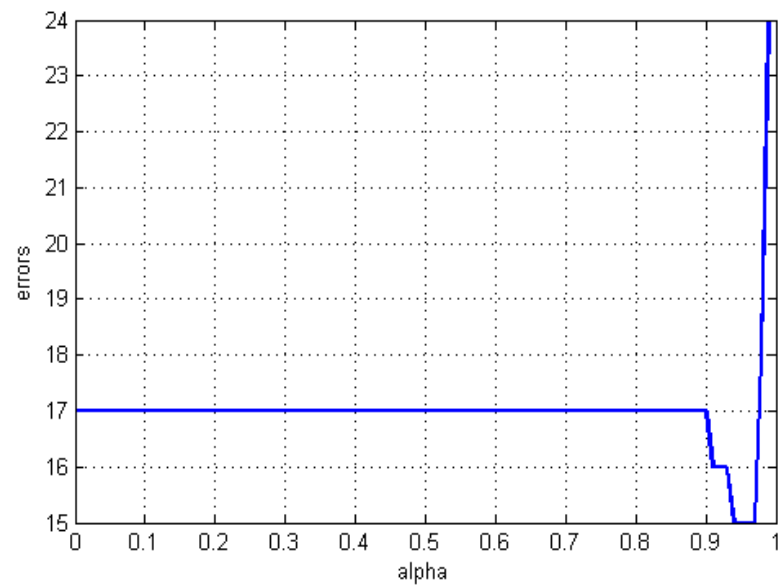

Figure 5. Clustering errors of standardized Iris data for Versicolor/Virginica varieties (50/50) with 15 misclassified objects.

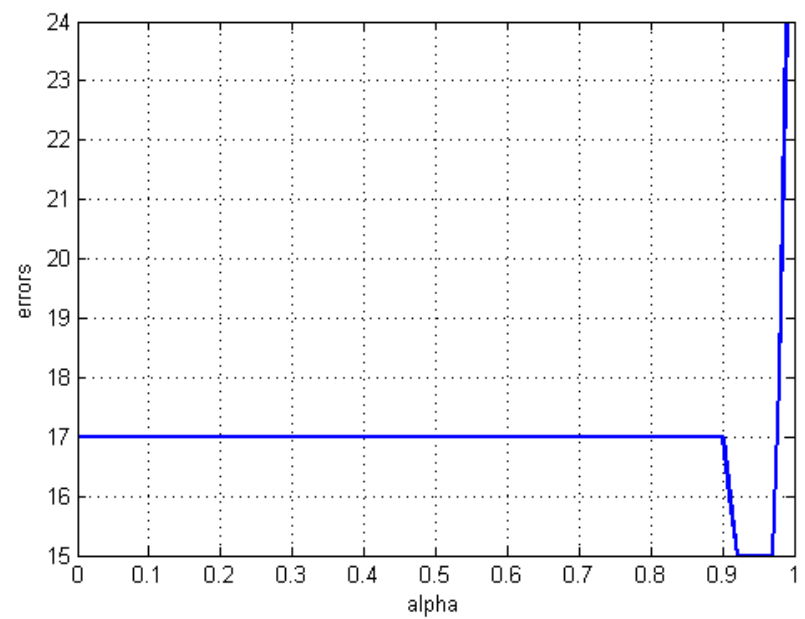

Figure 6. Clustering errors of standardized Iris data for Versicolor/Virginica varieties (70/30) with 15 misclassified objects. 


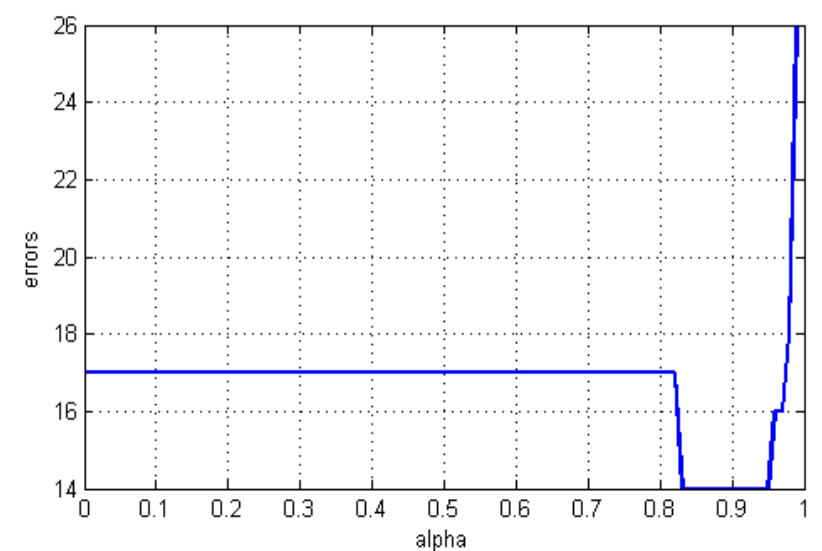

Figure 7. Clustering errors of standardized Iris data for Versicolor/Virginica varieties (30/70) with 14 misclassified objects.

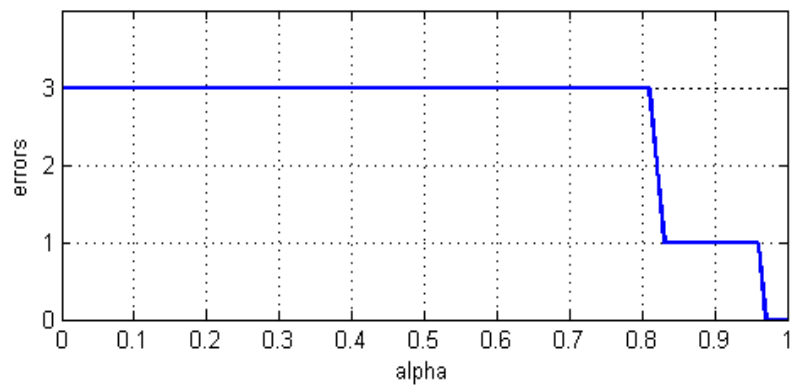

Figure 8: Clustering errors of original Iris data for Setosa versus Versicolor/Virginica varieties (50/100, 100/50, 30/120).

We reduce errors to zero (Fig. 8) and correctly separate the smaller $1^{\text {st }}$ class from the bigger one $\left(2^{\text {nd }}\right.$ and $\left.3^{\text {rd }}\right)$ in the optimal interval $0.97 \leq \alpha^{*} \leq 1$ for all initial partitions, i.e. $50 / 100,100 / 50,30 / 120$. For standardized data, the result contains no errors at all for the whole interval $0 \leq \alpha \leq 1$ for all initial partitions.

\section{Conclusion}

The $k$-means procedure is very popular in machine learning and data mining fields. This procedure is very natural and understanding its principles and results is easy. Additionally, this procedure is deeply connected with other ideas, like the EM-algorithm, SOMs, etc.

On the other hand, the use of the bi-partial criterion can improve the classical clustering result. The bi-partial objective function consists of two parts, the first one supporting the best approximation of individual categories, and the second one supporting the appropriate separation among the categories. In the case of the $k$-means algorithm, the bi-partial objective function combines intracluster dispersions with the inter-cluster similarity, to be jointly minimized. In dual form, the bi-partial objective function combines cluster concentrations with the intercluster dispersion, to be maximized.

In this paper, we investigate the direct form of the bipartial criterion function. The first part of this criterion provides the classical quality measure of $k$-means clustering, based on distances between objects.
As it is shown in this paper, the bi-partial criterion does not work directly through the standard procedure of the classical $k$-means, since the second part of the criterion cannot be changed within the classical procedure.

Therefore, to improve the clustering quality based on the bi-partial criterion, we develop here the new permutable version of the classical $k$-means algorithm.

As it is shown in this paper, the permutable $k$-means appears to be a new type of clustering procedures.

The permutable $k$-means uses distances and similarities only. Therefore, it does not need to use the featurebased representation of experimental data. To reduce the computational complexity of permutations we can use in further work the optimal iterative techniques.

It is easy to show that in the dual form the bi-partial objective function combines cluster concentrations with the inter-cluster dispersion, to be jointly maximized. The first part of both bi-partial objective functions provides the "standard" quality of clustering based on distances between objects (the classical $k$-means) or similarities between them in dual form (the similarity $k$-means).

As a result, what the algorithm have we built? It is clear, that we have merely shown the principle of developing a class of criteria and corresponding algorithms. As we can see in Figs. 1-7, error lines are not convex functions of $\alpha$ in general. The future study should, then, be oriented at defining conditions for convexity, on the one hand, and developing effective algorithms of extrema finding of the similar functions, on the other.

\section{Acknowledgements}

The work of the first author was supported by Russian Foundation for Basic Research under grant 17-07-00319.

\section{References}

[1] H. Steinhaus (1956). Sur la division des corps matériels en parties. Bulletin de l'Academie Polonaise des Sciences IV (C1.III), 801-804 (in French).

[2] M.I. Shlezinger (1965). Spontaneous discrimination of patterns. In: Reading Automata. Naukova Dumka, Kiev (in Russian).

[3] M.I. Shlezinger (1968). The interaction of learning and self-organization in pattern recognition. In: Kibernetika, 4(2), 81-88. http://irtc.org.ua/image/ Files/Schles/non-supervised.pdf

[4] A.V. Milen'kii (1975). Classification of signals in conditions of uncertainty. Moscow, Soviet Radio (in Russian).

[5] E. Diday et al. (1979). Optimisation en classification automatique. INRIA, Domaine de Voluceau, Rocquencourt B.P. 105, 78150 Le Chesnay (in French).

[6] W.S. Torgerson (1958). Theory and Methods of Scaling. N.Y., Wiley.

[7] H. P. Friedman and J. Rubin (1967). On Some Invariant Criteria for Grouping Data. In: J. of the American Statistical Association, 62(320):1159-1178. https://doi.org/10.1080/01621459.1967.10500923 
[8] H. Späth (1983). Cluster-formation und -analyse: Theorie, FORTRAN-Programme und Beispiele. R. Oldenbourg-Verlag, München - Wien.

[9] S.A. Aivazyan, et al. (1989). Applied Statistics. Classification and reduction of dimensionality (Ch. 5. Basic concepts and definitions used in classification without training. 5.4. Classification quality functionals and extremal approach to cluster analysis problems). Finansy i statistika, Moscow (in Russian)

[10] H.-J. Mucha, U. Simon, R. Brüggemann (2002). Model-based Cluster Analysis Applied to Flow Cytometry Data of Phytoplankton. Tech. Report, Berlin. http://www.wias-berlin.de/techreport/5/ wias_technicalreports_5.pdf

[11] E. Pekalska, R.P.W. Duin (2005). The Dissimilarity Representation for Pattern Recognition. Foundations and Applications. W.S. Singapore.

[12] A.W.F. Edwards, L.L. Cavalli-Sforza (1965). A Method for Cluster Analysis. In: Biometrics, 21, 362-375. https://www.jstor.org/stable/ 2528096 ?seq=1\#page_scan_tab_contents

[13] Jan W. Owsinski (2012). On the optimal division of an empirical distribution (and some related problems). In: Przeglad Statystyczny, special issue, 1, 109-122.

[14] Jan W. Owsinski (2013). On dividing an empirical distribution into optimal segments. http://new.sisstatistica.org/wp-content/uploads/ 2013/09/RS12On-Dividing-an-Empirical-Distribution-into.pdf

[15] Jan W. Owsinski (2011). The bi-partial approach in clustering and ordering: the model and the algorithms. In: Statistica \& Applicazioni. Special Issue, 43-59.
[16] Jan W. Owsinski (1990). On a new naturally indexed quick clustering method with a global objective function. In: Applied Stochastic Models and Data Analysis, 6(3), 157-171. https://doi.org/10.1002/asm.3150060303

[17] S.D. Dvoenko (2009). Clustering and separating of a set of members in terms of mutual distances and similarities. In: Transactions on MLDM. IBaI Publishing 2, 2 (Oct. 2009), 80-99.

[18] S. Dvoenko (2014). Meanless $k$-means as $k$-meanless clustering with the bi-partial approach. In: Proc. of $12^{\text {th }}$ Int. Conf. on Pattern Recognition and Image Processing (PRIP'2014). UIIP NASB, Minsk, Belarus, 50-54.

[19] R.O. Duda, P.E. Hart (1973). Pattern Classification and Scene Analysis. N.Y., Wiley.

[20] R.O. Duda, P.E. Hart, D.G.Stork (2000). Pattern Classification. Wiley-Interscience New York, NY.

[21] R.A. Fisher (1936). The use of multiple measurements in taxonomic problems. In Ann. Eugenics. 7, 2 (Sept. 1936), 179-188. https://doi.org/10.1111/j.1469-1809.1936.tb02137.x

[22] S. D. Dvoenko, D.O. Pshenichny (2016). A recovering of violated metric in machine learning. In: Proceedings of $8^{\text {th }}$ Int. Symposium on Information and Communication Technology (SoICT'2016). ACM NY, 15-21. https://doi.org/10.1145/3011077.3011084 